\title{
Chronic comorbidities and clinical outcomes in patients with and without COVID-19: a large population-based study using national administrative healthcare open data of Mexico
}

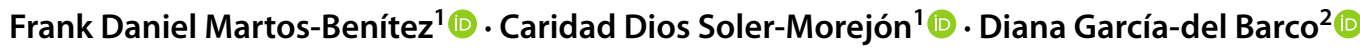

Received: 19 August 2020 / Accepted: 2 December 2020 / Published online: 7 January 2021

(c) Springer Nature Switzerland AG part of Springer Nature 2021

\begin{abstract}
Coronavirus disease 2019 (COVID-19) was rapidly expanded worldwide within a short period. Its relationship with chronic comorbidities is still unclear. We aimed to determine the effects of chronic comorbidities on clinical outcomes of patients with and without COVID-19. This was an analysis of 65,535 patients with suspicion of viral respiratory disease $(38,324$ SARS-CoV-2 positive and 27,211 SARS-CoV-2 negative) from January 01 to May 12, 2020 using the national administrative healthcare open data of Mexico. SARS-CoV-2 infection was confirmed by reverse-transcriptase-polymerase-chain-reaction. General characteristics and chronic comorbidities were explored. Clinical outcomes of interest were hospital admission, pneumonia, intensive care unit admission, endotracheal intubation and mortality. Prevalence of chronic comorbidities was 49.4\%. Multivariate logistic regression analysis showed that the effect of age, male sex, bronchial asthma, diabetes mellitus and chronic kidney disease on clinical outcomes was similar for both SARS-CoV-2 positive and negative patients. Adverse clinical outcomes were associated with the time from symptoms onset to medical contact, chronic obstructive pulmonary disease, hypertension and obesity in SARS-CoV-2 positive patients, but with cardiovascular disease in SARS-CoV-2 negative patients ( $p$ value $<0.01$ for all comparisons). Chronic comorbidities are commonly found in patients with suspicion of viral respiratory disease. The knowledge of the impact of comorbidities on adverse clinical outcomes can better define those COVID-19 patients at higher risk. The different impact of the specific type of chronic comorbidity on clinical outcomes in patients with and without SARS-CoV-2 infection requires further researches. These findings need confirmation using other data sources.
\end{abstract}

Keywords COVID-19 $\cdot$ Diabetes mellitus $\cdot$ Hypertension $\cdot$ Mortality $\cdot$ Obesity $\cdot$ SARS-CoV-2

\section{Introduction}

Coronavirus disease 2019 (COVID-19), a respiratory disease caused by the severe acute respiratory syndrome-coronavirus-2 (SARS-CoV-2), was rapidly expanded worldwide

Supplementary Information The online version contains supplementary material available at https://doi.org/10.1007/s1173 9-020-02597-5.

Frank Daniel Martos-Benítez

fdmartos@infomed.sld.cu

1 Intensive Care Unit 8B, Hermanos Ameijeiras Hospital, 701 San Lázaro St., Havana Centre, 10300 Havana City, Cuba

2 Biomedical Research Division, Centre for Genetic Engineering and Biotechnology, 25th St., Playa, 10300 Havana City, Cuba within a short period. The new SARS-CoV-2 coronavirus infection is clinically characterized by mild upper respiratory tract infection [1]; however, some cases may progress to aggressive viral pneumonia, acute respiratory failure and death [2].

SARS-CoV-2 coronavirus infection has become challenging for public health systems because of the high infection rates. It is crucial to understand the epidemiology and pathobiology of COVID-19 to reduce its impact. The rapid evolving pandemic requires sharing all information and instant experiences among healthcare workers in order to assess prognosis, risk stratification, allocation of healthcare resources and designing of clinical trials. Control strategies and isolation are necessary for prevention while innovative and costly therapies, including hospital beds and resources, are required for management. Implementing these measures requires considerable efforts by healthcare systems; 
thus, quantifying the influence of risk factors on clinical outcomes at the population level is critical. As for the literature revised, to date few analyses of this sort have been performed.

Since COVID-19 is a new infectious disease, its relationship with chronic comorbidities is still unclear. According to four systematic reviews and meta-analyses, chronic underlying diseases are highly prevalent among COVID-19 patients and are associated with severe cases [3-6]. Elderly people and those with chronic comorbidities such as respiratory, cardiovascular, metabolic, renal, and cancer have been associated with adverse clinical outcomes [6-11]. This study was aimed to determine the effects of chronic comorbidities on clinical outcomes of patients with and without COVID-19.

\section{Methods}

\section{Design, setting and patients}

The study was conducted in accordance with The REporting of studies Conducted using Observational Routinelycollected health Data (RECORD) Statement. This is a retrospective analysis of the national administrative healthcare open data from the General Directorate of Epidemiology of Mexico [12]. National administrative healthcare open data routinely collects information regarding origin, demography, epidemiology, chronic comorbidities and outcomes of patients with suspicion of viral respiratory disease, defined as cough, fever or headache last week more at least one of the following: dyspnoea, arthralgia, myalgia, sore throat, rhinorrhoea, conjunctivitis, or chest pain [13]. A complete list of codes used to classify chronic comorbidities, outcomes, and confounders is provided in Online Resource 1. The sentinel healthcare units of the System of Epidemiological Surveillance of Viral Respiratory Diseases, located at the first, second or third level of healthcare, transmitted the data to the institutional/ regional health authorities and then recorded in the SISVER platform once patients were registered in the medical unit. Quality of data was verified at each step. Patients with suspicion of viral respiratory disease were isolated and combined pharyngeal/ nasopharyngeal (for all patients), bronchoalveolar lavage (for intubated patients only), or lung biopsy (for deceased patients only) samples were collected for reverse-transcriptase-polymerase-chainreaction (RT-PCR) testing. RT-PCR testing was carried out by the National Network of Epidemiological Surveillance Laboratories, namely the Institute of Epidemiological Diagnosis and Reference "Dr Manuel Martínez Báez", State Laboratory of Public Health, and Support Laboratories for Epidemiological Surveillance; results were recorded in the SISVER platform [13]. Finally, data were summarized and published by the General Directorate of Epidemiology.
A total of 65,535 patients with suspicion of viral respiratory disease from 01 January to 13 May 2020 were analysed; of them, 38,324 were SARS-CoV-2 positive and 27 211 were SARS-CoV-2 negative. Flowchart of participants is depicted in Online Resource 2. Distribution of patients according to the sectors of the National Health System and regions of Mexico involved in the study is illustrated in Online Resource 3.

\section{Reverse-transcriptase-polymerase-chain-reaction testing}

RT-PCR testing for detecting SARS-CoV-2 virus was carried out as stated by the guidelines of the Institute of Epidemiological Diagnosis and Reference "Dr Manuel Martínez Báez" and endorsed by the General Directorate of Epidemiology of Mexico [20]. All types of RT-PCR tests had an analytical sensitivity of $\leq 250$ copies per reaction and analytical specificity of $100 \%$. As recommended by the World Health Organization, RT-PCR assays targeting the RNA-dependent RNA polymerase, envelope, and nucleocapsid genes of SARS-CoV-2 virus were used for $93.59 \%$ of patients (analytical sensitivity: 10 copies per reaction; analytical specificity: $100 \%$ ) [14]; other RT-PCR tests used by private laboratories (and listed in Online Resource 4) accounted for 5.45\%; unspecified type of RT-PCR tests was $0.96 \%$.

\section{General variables, chronic comorbidities and outcomes}

The following general data were examined: age, child/adult, biological sex, smoking habit, pregnancy, time from symptoms onset to medical contact, time from medical contact to death, and time from symptoms onset to death. Medical contact was defined as the date of the patient's registration into the medical record. The analysed chronic comorbidities were the following: chronic obstructive pulmonary disease (COPD), bronchial asthma, hypertension, cardiovascular disease, diabetes mellitus, obesity, chronic kidney disease, and others. The adverse clinical outcomes of interest were hospital admission, pneumonia, intensive care unit (ICU) admission, endotracheal intubation, and mortality.

\section{Statistical analysis}

Bias in detecting SARS-CoV-2 virus infection was unlikely since RT-PCR was achieved for all patients and was carried out by validated methods $[13,14]$. Study objective was unknown for healthcare physicians and patients, which allowed to minimize the following sources of biases: (1) selective report of chronic comorbidities and adverse clinical outcomes; and (2) selective mistake or unconscious omission in the report of adverse clinical outcomes. In this regard, the likelihood of 
mistake/ omission was the same for patients with and without chronic comorbidities. Finally, as national administrative healthcare open data might be another source of selection biases, we could expect that patients with SARS-CoV-2 infection were more likely to be reported; however, since patients with and without SARS-CoV-2 infection were separately analysed, this sort of bias was absolutely controlled.

The total of 38,324 SARS-CoV-2 positive and 27,211 SARS-CoV-2 negative patients reported in the national administrative healthcare open data were used for analysis. Variables registered in the dataset as "ignored" or "unspecified" were considered as missing data. As a small number of absent data was found (Online Resource 5), no treatment for missing data was carried out. Description of missing data was reported in the result section and not included in statistical analyses.

Categorical variables are shown as absolute numbers with percentage, whereas numerical variables are represented as means with standard deviation (SD) or medians with $25^{\text {th }}$ $-75^{\text {th }}$ intercuartil range (IQR) depending on whether the population was normally distributed (assessed by Kolmogorov-Smirnov test and Q-Q plot). Differences between groups were assessed using the chi-square test $\left(\chi^{2}\right)$ for categorical variables; $t$-test or Mann-Whitney $U$ test were used for continuous variables depending on whether the population was normally distributed or not, respectively.

Simple and multivariate logistic regression analyses were used to identify the factors associated with adverse clinical outcome. For each multivariate model, age, sex, smoking habit, time from symptoms onset to medical contact, and all the comorbidities were introduced. Classification tree with exhaustive CHAID method was used for categorizing numerical variables. Results were summarized as odds ratio (OR), 99\% confidence interval (CI), and $p$ value.

Two analyses were carried out. In the first, the total cohort of patients was examined. In the second, a subgroup analysis (for SARS-CoV-2 positive and for SARS-CoV-2 negative patients) was made.

Statistical tests with a two-tailed $p$ value $<0.01$ were considered as significant. Data were analysed using IBM®SPSS ${ }^{\circledR}$ Statistics 23.0 (IBM, Chicago, IL, USA). Microsoft Excel software was also used for graphs.

\section{Results}

\section{General characteristics of patients}

The mean age of the population studied was 44.0 years (SD 16.9 years), and $10,872(16.6 \%)$ patients had $>60$ years old. Male and female patients accounted for $53.2 \%$ and $46.8 \%$, respectively. Smoking habit was identified in 6,029 (9.2\%) patients. Pregnant women accounted for $1.0 \%$ of the total number of cases ( $2.2 \%$ of the female patients).
Children comprised $4.4 \%$ of patients. The median time from symptoms onset to medical contact was 3.0 days (IQR 1.0-6.0 days). Hospital and ICU admission was required in $32.3 \%$ and $2.9 \%$ of patients, respectively. Pneumonia was diagnosed in $22.7 \%$, endotracheal intubation was used in $2.6 \%$, and overall mortality rate was $6.7 \%$. The median time from symptoms onset to death and time from medical contact to death was 9.0 days (IQR 6.0-13.0 days) and 5.0 days (IQR 2.0-9.0 days), respectively. Table 1 illustrates the general characteristics of patients.

\section{Chronic comorbidities}

Chronic comorbidities were found in 28,668 patients (43.7\%); of them, 7,780 (27.1\%) had two comorbidities and $4,122(14.4 \%)$ had three or more comorbidities. The prevalence of chronic comorbidities in both SARS-CoV-2 positive and negative patients was $46.9 \%$ and $39.4 \%$, respectively. The most common diseases were hypertension, obesity and diabetes mellitus (Fig. 1a and b).

Among SARS-CoV-2 positive patients, age in patients with chronic comorbidities was higher than that in patients without comorbidities (52.2 vs. 42.0 years; $p<0.0001$ ); additionally, differences in distribution of time from symptoms onset to medical contact ( $p=0.003)$, time from medical contact to death $(p<0.0001)$ and time from symptoms onset to death $(p<0.0001)$ were observed between patients with and without chronic comorbidities. In SARS-CoV-2 negative group, patients with chronic comorbidities were older than those without chronic comorbidities (mean 47.6 vs. 34.8 years; $p<0.0001$ ); however, time from symptoms onset to medical contact ( $p=0.149)$, time from symptoms onset to death $(p=0.021)$ and time from medical contact to death $(p=0.184)$ in patients with chronic comorbidities were similar to those in patients without chronic comorbidities.

\section{Factors associated with adverse clinical outcomes in the total cohort}

Univariate analysis of factors associated with adverse clinical outcomes in the total cohort is depicted in Online Resource 6. Multivariate analyses demonstrated that age, sex male, time from symptoms onset to medical contact, numerous chronic comorbidities, and SARS-CoV-2 positive were independently associated with several adverse clinical outcomes (Table 2). Noteworthy, SARS-CoV-2 positive and age $\geq 80$ years had the greatest size of effect. 
Table 1 General characteristics of participants

\begin{tabular}{|c|c|c|c|c|}
\hline \multirow[t]{2}{*}{ Variables } & \multicolumn{2}{|c|}{$\begin{array}{l}\text { SARS-CoV-2 positive } \\
n=38,324\end{array}$} & \multicolumn{2}{|c|}{$\begin{array}{l}\text { SARS-CoV-2 negative } \\
n=27,211\end{array}$} \\
\hline & $n(\%)$ & $\begin{array}{l}\text { Mean (SD) or } \\
\text { median (IQR) }\end{array}$ & $n(\%)$ & $\begin{array}{l}\text { Mean (SD) or } \\
\text { median (IQR) }\end{array}$ \\
\hline Age, years; mean (SD) & - & $46.9(15.7)$ & - & $39.9(17.6)$ \\
\hline \multicolumn{5}{|l|}{ Age, years; $n(\%)$} \\
\hline$\leq 20$ & $955(2.5)$ & - & $2570(9.4)$ & - \\
\hline $21-40$ & $13,220(34.5)$ & - & $12,688(46.6)$ & - \\
\hline $41-60$ & $16,662(43.5)$ & - & $8568(31.5)$ & - \\
\hline $61-80$ & $6632(17.3)$ & - & $2810(10.3)$ & - \\
\hline$\geq 80$ & $855(2.2)$ & - & $575(2.1)$ & - \\
\hline Sex, $n(\%)$ & & - & & - \\
\hline Male & $22,362(58.3)$ & & $12,531(46.0)$ & \\
\hline Female & $15,962(41.7)$ & & $14,680(54.0)$ & \\
\hline Smoking habit, $n(\%)$ & $3277(8.6)$ & - & $2752(10.1)$ & - \\
\hline Pregnant, $n(\%)$ & $239(0.62)$ & - & $441(1.6)$ & - \\
\hline Children, $n(\%)$ & $731(1.9)$ & - & $2168(8.0)$ & - \\
\hline Time from symptoms onset to medical contact, days; median (IQR) & - & $4.0(2.0-6.0)$ & - & $3.0(1.0-5.0)$ \\
\hline \multicolumn{5}{|l|}{ Time from symptoms onset to medical contact, days; $n(\%)$} \\
\hline $0-3$ & $18,175(47.4)$ & - & $17,047(62.6)$ & - \\
\hline $4-7$ & $15,146(39.5)$ & - & $7679(28.2)$ & - \\
\hline$\geq 8$ & $5003(13.1)$ & - & $2485(9.1)$ & - \\
\hline Hospital admission, $n(\%)$ & $15,305(39.9)$ & - & $5891(21.6)$ & - \\
\hline Missing, $n(\%)$ & $0(0.0)$ & & $0(0.0)$ & \\
\hline Pneumonia, $n(\%)$ & $11,501(30.0)$ & - & $3,367(12.4)$ & - \\
\hline Missing, $n(\%)$ & $3(0.0078)$ & & $0(0.0)$ & \\
\hline ICU admission, $n(\%)$ & $1492(3.9)$ & - & $382(1.4)$ & - \\
\hline Missing, $n(\%)$ & $0(0.0)$ & & $0(0.0)$ & \\
\hline Endotracheal intubation, $n(\%)$ & $1534(4.0)$ & - & $194(0.71)$ & - \\
\hline Missing, $n(\%)$ & $0(0.0)$ & & $0(0.0)$ & \\
\hline Mortality, $n(\%)$ & $3926(10.2)$ & - & $477(1.8)$ & - \\
\hline Missing, $n(\%)$ & $0(0.0)$ & & $0(0.0)$ & \\
\hline Time from symptoms onset to mortality, days; median (IQR) & - & $9.0(6.0-14.0)$ & - & $6.0(3.0-10.0)$ \\
\hline Time from medical contact to mortality, days; median (IQR) & - & $5.0(2.0-9.0)$ & - & $3.0(1.0-7.0)$ \\
\hline
\end{tabular}

$I C U$ intensive care unit; $I Q R$ 25th-75th intercuartil range; $S D$ standard deviation

\section{Factors associated with adverse clinical outcomes by subgroup analysis}

The rates of adverse clinical outcomes were significantly higher in patients with chronic comorbidities than those in patients without chronic comorbidities for both SARS-CoV-2 positive and negative subgroups, as shown by univariate analysis (Online Resource 7, 8, 9, 10, 11). Multivariate logistic regression analysis demonstrated that age $>40$ years, sex male, time from symptoms onset to medical contact $>3$ days, and chronic comorbidities such as COPD, hypertension, diabetes mellitus, obesity and chronic kidney disease were independent risk factors for adverse clinical outcomes in SARS-CoV-2 positive patients (Table 3). For SARS-CoV-2 negative patients, age $>60$ years, sex male and chronic comorbidities such as cardiovascular disease, diabetes mellitus and chronic kidney disease were the risk factors associated with an increased risk of adverse clinical outcomes (Table 4).

\section{Discussion}

Since December 2019 until present, more than 1 million people have died globally as a result of COVID-19 [15]; furthermore, COVID-19 pandemic has strained the medical systems worldwide and new outbreaks have been reported in 


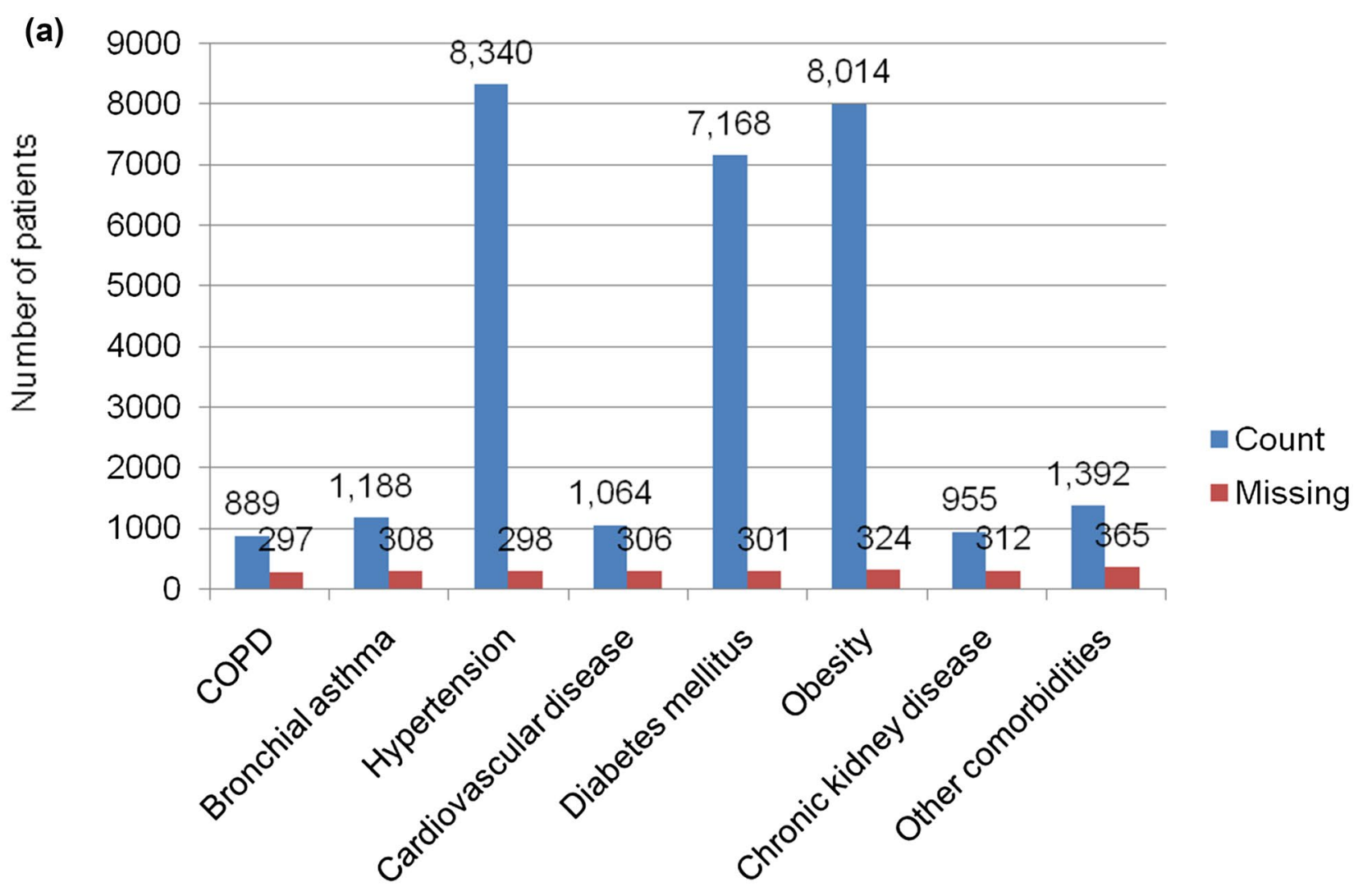

(b)

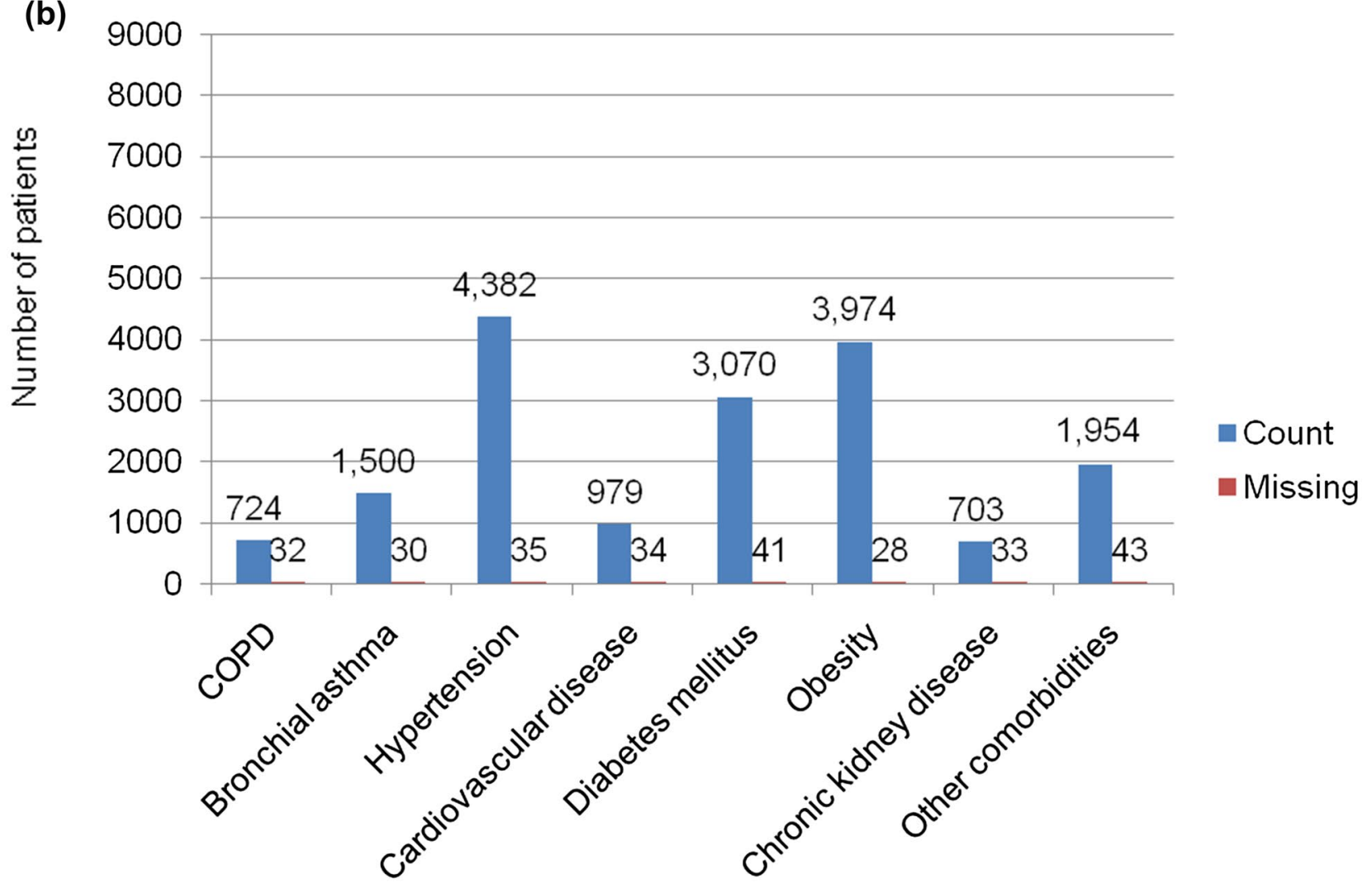

Fig. 1 Distribution of chronic comorbidities in SARS-CoV-2 positive (a) and negative (b) patients. COPD chronic obstructive pulmonary disease 
Table 2 Multivariate logistic regression analyses of factors associated with adverse clinical outcomes in the total cohort

\begin{tabular}{|c|c|c|c|c|c|}
\hline Variables & $\begin{array}{l}\text { Hospital admission } \\
\text { OR }(99 \% \mathrm{CI}) \\
p \text { value }\end{array}$ & $\begin{array}{l}\text { Pneumonia } \\
\text { OR }(99 \% \mathrm{CI}) \\
p \text { value }\end{array}$ & $\begin{array}{l}\text { ICU admission } \\
\text { OR }(99 \% \mathrm{CI}) \\
p \text { value }\end{array}$ & $\begin{array}{l}\text { Endotracheal intubation } \\
\text { OR }(99 \% \mathrm{CI}) \\
p \text { value }\end{array}$ & $\begin{array}{l}\text { Mortality } \\
\text { OR }(99 \% \mathrm{CI}) \\
p \text { value }\end{array}$ \\
\hline \multicolumn{6}{|l|}{ Age, years } \\
\hline$\leq 20$ & Ref. & Ref. & Ref. & Ref. & Ref. \\
\hline $21-40$ & $0.30(0.27-0.34)^{* *}$ & $0.45(0.39-0.51)^{* *}$ & $0.31(0.22-0.43) * *$ & $0.44(0.27-0.71)^{* *}$ & $0.58(0.40-0.86)^{* *}$ \\
\hline $41-60$ & $0.70(0.63-0.78)^{* *}$ & $0.99(0.87-1.14)$ & $0.77(0.56-1.06)$ & $1.27(0.80-2.00)$ & $2.16(1.49-3.12)^{* *}$ \\
\hline $61-80$ & $1.75(1.55-1.97)^{* *}$ & $1.96(1.70-2.25)^{* *}$ & $1.38(0.99-1.92)$ & $2.57(1.61-4.10)^{* *}$ & $4.88(3.36-7.08)^{* *}$ \\
\hline$\geq 80$ & $2.60(2.15-3.14)^{* *}$ & $2.79(2.30-3.39)^{* *}$ & $1.55(1.01-2.37)^{*}$ & $2.43(1.40-4.23)^{* *}$ & $7.22(4.81-10.84)^{* *}$ \\
\hline Sex, male & $1.62(1.54-1.70)^{* *}$ & $1.57(1.49-1.66)^{* *}$ & $1.64(1.43-1.87)^{* *}$ & $1.69(1.47-1.96)^{* *}$ & $1.67(1.52-1.83)^{* *}$ \\
\hline Smoking habit & $1.01(0.93-1.10)$ & $1.03(0.94-1.13)$ & $0.93(0.75-1.16)$ & $1.07(0.86-1.33)$ & $0.93(0.80-1.08)$ \\
\hline \multicolumn{6}{|c|}{$\begin{array}{l}\text { Time from symptoms onset } \\
\text { to medical contact, days }\end{array}$} \\
\hline $0-3$ & Ref. & Ref. & Ref. & Ref. & Ref. \\
\hline $4-7$ & $0.95(0.90-1.00)$ & $1.32(1.24-1.40)^{* *}$ & $1.33(1.16-1.52)^{* *}$ & $1.45(1.26-1.67)^{* *}$ & $1.10(1.00-1.21)^{*}$ \\
\hline$\geq 8$ & $1.01(0.93-1.09)$ & $1.41(1.30-1.53)^{* *}$ & $1.34(1.11-1.62)^{* *}$ & $1.35(1.11-1.65)^{* *}$ & $0.95(0.83-1.09)$ \\
\hline COPD & $1.90(1.63-2.23)^{* *}$ & $1.56(1.34-1.80)^{* *}$ & $1.04(0.76-1.42)$ & $1.11(0.81-1.53)$ & $1.47(1.21-1.78)^{* *}$ \\
\hline Bronchial asthma & $0.79(0.69-0.91)^{* *}$ & $0.85(0.73-0.99)^{*}$ & $0.89(0.61-1.28)$ & $0.53(0.32-0.87)^{*}$ & $0.81(0.62-1.07)$ \\
\hline High blood pressure & $1.26(1.18-1.34)^{* *}$ & $1.21(1.13-1.29)^{* *}$ & $1.20(1.03-1.40)^{*}$ & $1.14(0.98-1.33)$ & $1.32(1.19-1.46)^{* *}$ \\
\hline Cardiovascular disease & $1.34(1.17-1.54)^{* *}$ & $1.23(1.07-1.41)^{* *}$ & $1.31(1.00-1.70)^{*}$ & $1.22(0.92-1.62)$ & $1.17(0.96-1.40)$ \\
\hline Diabetes mellitus & $2.18(2.04-2.33)^{* *}$ & $1.83(1.71-1.96)^{* *}$ & $1.51(1.30-1.75)^{* *}$ & $1.51(1.30-1.76)^{* *}$ & $1.73(1.57-1.91)^{* *}$ \\
\hline Obesity & $1.28(1.20-1.36)^{* *}$ & $1.31(1.23-1.40)^{* *}$ & $1.48(1.28-1.71)^{* *}$ & $1.64(1.42-1.90)^{* *}$ & $1.49(1.35-1.65)^{* *}$ \\
\hline Chronic kidney disease & $3.29(2.79-3.87)^{* *}$ & $1.83(1.58-2.12)^{* *}$ & $1.25(0.93-1.69)$ & $1.44(1.07-1.94)^{*}$ & $2.19(1.83-2.63)^{* *}$ \\
\hline Other comorbidities & $1.51(1.35-1.68)^{* *}$ & $1.29(1.14-1.45)^{*}$ & $1.40(1.09-1.81)^{*}$ & $0.83(0.59-1.18)$ & $1.53(1.27-1.84)^{* *}$ \\
\hline SARS-CoV-2 positive & $2.15(2.04-2.27) * *$ & $2.52(2.38-2.68)^{* *}$ & $2.20(1.88-2.58)^{* *}$ & $4.04(3.29-4.97)^{* *}$ & $5.23(4.57-5.98)^{* *}$ \\
\hline
\end{tabular}

Missing cases were not included in analysis

$C I$ confidence interval; $I C U$ intensive care unit; $O R$ odds ratio; Ref. reference category

${ }^{*} p$ value $<0.01 ; * * p$ value $<0.001$

several regions including Europe [15]. We found that SARSCoV-2 infection and age $\geq 80$ years had the greatest size of effect, demonstrating that COVID-19 and oldest patients had the most powerful association with worse clinical outcomes at the population level. These results support that the persistent cries of alarm is imperative because the world remains in danger.

Age was related to adverse clinical outcomes in both SARS-CoV-2 positive and negative patients, but the age at risk was lower for COVID-19 patients (40 vs. 60 years). Although age has been repeatedly related to adverse clinical outcomes in previous studies of COVID-19 [8, 16-19], the present results show that the strength of association could be greater than believed. Immune response to viral infections is reduced over time because of a limited cross-reactivity between anti-seasonal coronavirus and anti-SARS antibodies. In previously exposed individuals to seasonal coronaviruses but waning antibody titres (e.g. the elderly), the recall antibody production after SARS-CoV-2 infection can results in a pro-inflammatory state [20].
Among patients with SARS-CoV-2 infection, the time from symptoms onset to medical contact was found as a factor related to adverse clinical outcomes, highlighting the importance of an early medical seeking. General availability of health coverage and population-educating programs should be implemented in order to avoid delayed medical care. We found that sex male increased the risk of adverse clinical outcomes. Other studies reported similar results [18, $19,21]$. Immunity system is weaker in male than in female sex, therefore men are at higher risk of viral infection than women [22]. Because the low prevalence of smoking in patients hospitalized with COVID-19 [23, 24], the protective effect of tobacco is largely discussed [25]; however, several meta-analyses of observational studies have confirmed that smoking habit is related to severe COVID-19 and adverse outcomes [19, 21, 26-28]. Tobacco smoking is associated with upregulation of the angiotensin-converting-enzyme-2 (ACE2), immune system disturbance, endothelial injury and hypercoagulable state [28, 29]; these factors and pre-existing comorbidities could explain adverse outcomes observed in smokers with COVID-19. We found no effect of smoking 
Table 3 Multivariate logistic regression analyses of factors associated with adverse clinical outcomes in the SARS-CoV-2 positive patients

\begin{tabular}{|c|c|c|c|c|c|}
\hline Chronic comorbidities & $\begin{array}{l}\text { Hospital admission } \\
\text { OR }(99 \% \text { CI })\end{array}$ & $\begin{array}{l}\text { Pneumonia } \\
\text { OR }(99 \% \mathrm{CI})\end{array}$ & $\begin{array}{l}\text { ICU admission } \\
\text { OR }(99 \% \mathrm{CI})\end{array}$ & $\begin{array}{l}\text { Endotracheal intubation } \\
\text { OR }(99 \% \mathrm{CI})\end{array}$ & $\begin{array}{l}\text { Mortality } \\
\text { OR }(99 \% \text { CI })\end{array}$ \\
\hline \multicolumn{6}{|l|}{ Age, years } \\
\hline$\leq 20$ & Ref. & Ref. & Ref. & Ref. & Ref. \\
\hline $21-40$ & $1.06(0.84-1.34)$ & $1.32(1.00-1.74)$ & $0.59(0.32-1,11)$ & $0.83(0.38-1.81)$ & $1.57(0.75-3.28)$ \\
\hline $41-60$ & $2.75(2.19-3.46)^{* *}$ & $3.01(2.28-3.97)^{* *}$ & $1.59(0.86-2.92)$ & $2.43(1.14-5.20)^{*}$ & $5.97(2.90-12.33)^{* *}$ \\
\hline $61-80$ & $6.13(4.84-7.77)^{* *}$ & $5.50(4.14-7.30)^{* *}$ & $2.74(1.47-5.07)^{* *}$ & $4.78(2.23-10.27)^{* *}$ & $13.47(6.51-27.85)^{* *}$ \\
\hline$>80$ & $7.18(5.32-9.70)^{* *}$ & $7.09(5.09-9.88)^{* *}$ & $3.05(1.53-6.11)^{* *}$ & $4.47(1.94-10.27)^{* *}$ & $20.44(9.66-43.26)^{* *}$ \\
\hline Sex, male & $1.71(1.61-1.82)^{* *}$ & $1.58(1.48-1.68)^{* *}$ & $1.64(1.41-1.91)^{* *}$ & $1.76(1.51-2.06)^{* *}$ & $1.70(1.54-1.88)^{* *}$ \\
\hline Smoking habit & $0.97(0.87-1.08)$ & $1.05(0.94-1.17)$ & $0.87(0.68-1.12)$ & $1.01(0.79-1.27)$ & $0.89(0.76-1.05)$ \\
\hline \multicolumn{6}{|c|}{$\begin{array}{l}\text { Time from symptoms onset } \\
\text { to medical contact, days }\end{array}$} \\
\hline $0-3$ & Ref. & Ref. & Ref. & Ref. & Ref. \\
\hline $4-7$ & $1.14(1.07-1.22)^{* *}$ & $1.47(1.38-1.58)^{* *}$ & $1.47(1.26-1.71)^{* *}$ & $1.46(1.25-1.70)^{* *}$ & $1.14(1.03-1.26)^{*}$ \\
\hline$\geq 8$ & $1.16(1.06-1.27)^{* *}$ & $1.54(1.40-1.69)^{* *}$ & $1.39(1.13-1.72)^{* *}$ & $1.36(1.10-1.68)^{* *}$ & $0.95(0.82-1.09)$ \\
\hline COPD & $1.70(1.35-2.05)^{* *}$ & $1.29(1.07-1.56)^{*}$ & $1.18(0.83-1.68)$ & $1.18(0.84-1.67)$ & $1.50(1.21-1.87)^{* *}$ \\
\hline Bronchial asthma & $0.71(0.59-0.85)^{* *}$ & $0.78(0.64-0.94)^{*}$ & $1.00(0.65-1.54)$ & $0.54(0.31-0.95)^{*}$ & $0.86(0.64-1.16)$ \\
\hline High blood pressure & $1.27(1.18-1.37)^{* *}$ & $1.22(1.13-1.32)^{* *}$ & $1.20(1.02-1.41)^{*}$ & $1.13(0.96-1.33)$ & $1.33(1.20-1.48)^{* *}$ \\
\hline Cardiovascular disease & $1.08(0.90-1.30)$ & $1.03(0.86-1.22)$ & $1.02(0.72-1.43)$ & $1.03(0.74-1.44)$ & $1.04(0.84-1.30)$ \\
\hline Diabetes mellitus & $2.11(1.94-2.28)^{* *}$ & $1.80(1.66-1.95)^{* *}$ & $1.43(1.21-1.68)^{* *}$ & $1.46(1.25-1.72)^{* *}$ & $1.68(1.52-1.87)^{* *}$ \\
\hline Obesity & $1.35(1.25-1.45)^{* *}$ & $1.38(1.28-1.49)^{* *}$ & $1.49(1.28-1.75)^{* *}$ & $1.58(1.35-1.85)^{* *}$ & $1.53(1.38-1.71)^{* *}$ \\
\hline Chronic kidney disease & $2.58(2.08-3.20)^{* *}$ & $1.66(1.38-2.00)^{* *}$ & $1.09(0.76-1.57)$ & $1.32(0.94-1.85)$ & $2.18(1.77-2.69) * *$ \\
\hline Other comorbidities & $1.30(1.11-1.52)^{* *}$ & $1.14(0.97-1.35)$ & $1.19(0.85-1.68)$ & $0.75(0.49-1.12)$ & $1.29(1.04-1.61)^{*}$ \\
\hline
\end{tabular}

Missing cases were not included in analysis

$C I$ confidence interval; $C O P D$ chronic obstructive pulmonary disease; ICU intensive care unit; $O R$ odds ratio

${ }^{*} p$ value $<0.01 ; * *$ value $<0.001$

habit on clinical outcomes, which could be partially due to the aforementioned low prevalence of smoking in COVID19 patients, misclassification of smokers as nonsmokers, and the composition of the study participants: most previous studies have been focused on hospitalized patients while we carried out a population-based study including out-hospital patients. Our results are placed in the middle region of the risk spectrum: no protective or harmful effect, which increases the controversy on this topic. According to the currently contradictory knowledge, further large prospective studies are required to clarify the relationship between smoking habit and COVID-19.

We found the prevalence of respiratory diseases, hypertension, cardiovascular diseases, obesity and chronic kidney disease in COVID-19 patients was similar to those described in previous studies $[3,6,8,10]$; nonetheless, the prevalence of diabetes mellitus was slightly higher than those reported by other authors $[6,9]$, which may be explained by the variation in the prevalence of chronic comorbidities among different countries. As previous studies in COVID19 patients [4, 6, 8-10, 16-18], we observed a relationship between chronic comorbidities (e.g. COPD, hypertension, diabetes mellitus, obesity and chronic kidney disease) and adverse clinical outcomes. This association could be expected because patients with chronic diseases are usually aged, immunosenescent, or have impaired respiratory tract function. Particularly, COVID-19 pandemic have been challenged in countries with a high prevalence of obesity [30]; morbidity and mortality rates are higher in individuals with obesity and COVID-19 [31, 32]. Explanations for this association include the chronic pro-inflammatory state, the excessive oxidative stress response, and the impaired immunity; cytokines, mammalian target of rapamycin, and altered natural killer cell also play a central role [33]. Preventive weight-loss measures should be stimulated in obese patients.

Bronchial asthma was not associated with clinical outcomes in our analysis; other studies have shown similar results [34-36]. Type 2 immune response and its related cytokines such as IL-4 and IL-13, accumulation of eosinophils, and conventional therapeutics (e.g. inhaled corticosteroids, allergen immunotherapy, and anti-IgE monoclonal antibody) might reduce the risks of asthmatics suffering viral infection through alleviating inflammation or enhancing antiviral defence, which provides potential protective effects against COVID-19 [37]. Because the effect of bronchial asthma, diabetes mellitus and chronic kidney disease on 
Table 4 Multivariate logistic regression analyses of factors associated with adverse clinical outcomes in the SARS-CoV-2 negative patients

\begin{tabular}{|c|c|c|c|c|c|}
\hline Chronic comorbidities & $\begin{array}{l}\text { Hospital admission } \\
\text { OR }(99 \% \text { CI })\end{array}$ & $\begin{array}{l}\text { Pneumonia } \\
\text { OR }(99 \% \mathrm{CI})\end{array}$ & $\begin{array}{l}\text { ICU admission } \\
\text { OR }(99 \% \text { CI })\end{array}$ & $\begin{array}{l}\text { Endotracheal intubation } \\
\text { OR }(99 \% \mathrm{CI})\end{array}$ & $\begin{array}{l}\text { Mortality } \\
\text { OR }(99 \% \text { CI })\end{array}$ \\
\hline \multicolumn{6}{|l|}{ Age, years } \\
\hline$\leq 20$ & Ref. & Ref. & Ref. & Ref. & Ref. \\
\hline $21-40$ & $0.20(0.18-0.23)^{* *}$ & $0.28(0.24-0.33)^{* *}$ & $0.23(0.14-0.36)^{* *}$ & $0.20(0.10-0.42)^{* *}$ & $0.29(0.17-0.50)^{* *}$ \\
\hline $41-60$ & $0.34(0.30-0.40)^{* *}$ & $0.54(0.46-0.64)^{* *}$ & $0.42(0.27-0.65)^{* *}$ & $0.42(0.21-0.84)^{*}$ & $0.84(0.52-1.36)$ \\
\hline $61-80$ & $1.19(1.01-1.41)^{*}$ & $1.38(1.13-1.67)^{* *}$ & $0.86(0.52-1.41)$ & $1.16(0.56-2.39)$ & $1.95(1.17-3.25)^{*}$ \\
\hline$>80$ & $2.42(1.81-3.23)^{* *}$ & $2.10(1.58-2.78)^{* *}$ & $0.98(0.48-1.97)$ & $1.12(0.42-3.01)$ & $2.63(1.41-4.90)^{* *}$ \\
\hline Sex, male & $1.38(1.26-1.50)^{* *}$ & $1.46(1.32-1.62)^{* *}$ & $1.53(1.16-2.03)^{* *}$ & $1.24(0.84-1.83)$ & $1.41(1.10-1.82)^{*}$ \\
\hline Smoking habit & $1.13(0.98-1.30)$ & $1.03(0.88-1.22)$ & $1.19(0.78-1.80)$ & $1.51(0.89-2.56)$ & $1.23(0.86-1.76)$ \\
\hline \multicolumn{6}{|c|}{$\begin{array}{l}\text { Time from symptoms onset } \\
\text { to medical contact, days }\end{array}$} \\
\hline $0-3$ & Ref. & Ref. & Ref. & Ref. & Ref. \\
\hline $4-7$ & $0.59(0.53-0.66)^{* *}$ & $0.95(0.84-1.06)$ & $0.87(0.63-1.22)$ & $1.39(0.91-2.13)$ & $0.81(0.60-1.10)$ \\
\hline$\geq 8$ & $0.76(0.65-0.89)^{* *}$ & $1.15(0.97-1.37)$ & $1.26(0.81-1.97)$ & $1.31(0.68-2.51)$ & $1.12(0.74-1.70)$ \\
\hline COPD & $2.18(1.71-2.78)^{* *}$ & $1.94(1.54-2.44)^{* *}$ & $0.71(0.37-1.35)$ & $0.80(0.37-1.74)$ & $1.38(0.90-2.12)$ \\
\hline Bronchial asthma & $0.92(0.76-1.12)$ & $1.00(0.80-1.26)$ & $0.69(0.33-1.45)$ & $0.49(0.15-1.60)$ & $0.64(0.32-1.28)$ \\
\hline High blood pressure & $1.26(1.12-1.42)^{* *}$ & $1.20(1.05-1.38)^{*}$ & $1.24(0.86-1.79)$ & $1.32(0.81-2.14)$ & $1.26(0.92-1.71)$ \\
\hline Cardiovascular disease & $1.69(1.37-2.08)^{* *}$ & $1.53(1.24-1.88)^{* *}$ & $2.00(1.29-3.08)^{* *}$ & $1.93(1.10-3.39)^{*}$ & $1.64(1.13-2.39)^{*}$ \\
\hline Diabetes mellitus & $2.36(2.08-2.69)^{* *}$ & $1.87(1.62-2.16)^{* *}$ & $1.93(1.34-2.77)^{* *}$ & $1.95(1.21-3.16)^{* *}$ & $2.21(1.63-2.99)^{* *}$ \\
\hline Obesity & $1.08(0.95-1.22)$ & $1.08(0.94-1.24)^{* *}$ & $1.37(0.97-1.95)$ & $2.09(1.35-3.24)^{* *}$ & $1.16(0.85-1.59)$ \\
\hline Chronic kidney disease & $4.65(3.61-5.98)^{* *}$ & $2.14(1.69-2.72)^{* *}$ & $1.63(0.98-2.72)$ & $1.87(0.99-3.55)$ & $2.23(1.50-3.31)^{* *}$ \\
\hline Other comorbidities & $1.74(1.50-2.02)^{* *}$ & $1.47(1.24-1.74)^{* *}$ & $1.81(1.22-2.69)^{* *}$ & $1.14(0.60-2.15)$ & $2.44(1.75-3.39)^{* *}$ \\
\hline
\end{tabular}

Missing cases were not included in analysis

$C I$ confidence interval; $C O P D$ chronic obstructive pulmonary disease; ICU intensive care unit; $O R$ odds ratio

${ }^{*} p$ value $<0.01 ; * *$ value $<0.001$

clinical outcomes was similar for both SARS-CoV-2 positive and negative patients, it may represent the impact of these chronic comorbidities on the health status of general population; indeed, some meta-analyses have failed to demonstrate a pooled effect of chronic kidney disease [4] and diabetes mellitus on severity of COVID-19 [6]. Cardiovascular disease has been linked to poor clinical outcomes in COVID-19 patients [19, 21], but such association was not found in the present study. Potential reasons for this unexpected result are the study population (other researches have been centred in hospitalized or critically ill patients while we carried out a population-based study including out-hospital patients), the number and type of covariates included in multivariate analysis (we analysed the covariates with all chronic comorbidities, therefore all patients' information was explored and taken as a whole, depicting the real clinical context), the definition of cardiovascular disease used in specific studies, and the random characteristics of the study population. Conversely, COVID-19 patients with COPD, hypertension, or obesity seem to have a real increased risk for adverse clinical outcomes. These results suggest a different impact of the specific type of chronic comorbidity on clinical outcomes between patients with and without SARS-CoV-2 infection, which requires further researches.

According to the current knowledge, the most important pathophysiological factors in COVID-19 seem to be the viral load and the patient's immune response as described before $[38,39]$; then chronic comorbidities might modify the magnitude of the response to viral aggression by amplifying the inflammatory pathways [40]. The loss of ACE2-mediated protection against the pro-inflammatory, vasoconstrictor and pro-fibrotic effects of angiotensin II [41]; endothelial dysfunction and pro-thrombotic state [42]; and single nucleotide polymorphisms in the host DNA might contribute to worse clinical outcomes among COVID-19 patients with chronic comorbidities [43].

COVID-19 patients with chronic underlying diseases are high-risk patients requiring strict adherence to preventive and treatment protocols. This approach supports those interventions aimed to reduce the viral load (e.g. preventive strategies including vaccination program, antiviral therapies, plasma from convalescent patients) and immunological reinforcement (e.g. type I interferon, innate immunity enhancement approaches). Since the COVID-19 pandemic remains as a real world threat [15], ICU practitioners, hospital 
administrators, governments, and policy makers must prepare for an increase in hospital and ICU beds requirements; quantifying the level of risk related to chronic underlying diseases is vital for these purposes. This information may help the healthcare systems to guide vulnerable populations, assess the risk of deterioration, organize the human and material health resources, and optimize the health cost.

There are several strengths in this study. First, this is an adequately powered study which includes a large number of patients. Second, this is a large population-based study assessing the influence of a variety of specific chronic underlying diseases on several clinical outcomes in patients with COVID-19, which depicts the real clinical context. Most previous studies used small sample sizes, single centre observations, heterogeneous data, and usually focused on just one clinical outcome [6-11]; these limitations may lead to biases and uncertain statistical estimations, which were overcome through this large population-based study. Third, we also studied the clinical impact of chronic comorbidities among patients without COVID-19, which has not been performed before. These data account for a holistic analysis about the real impact of chronic underlying diseases for healthcare systems in the current setting of respiratory diseases and COVID-19 pandemic.

Limitations of this study should be addressed. First, this is a retrospective analysis where chronic underlying diseases were self-reported. Underreport of chronic comorbidities could modify the estimation of the strength of association with clinical outcomes; however, the prevalence of chronic comorbidities was similar to those informed in other cohorts of COVID-19 patients [3, 6, 9, 10]. Second, some patients with pneumonia in the SARS-CoV-2 negative group might actually have a falsely negative RT-PCR in the upper respiratory tract sample. Bronchoalveolar lavage by bronchoscopy, a method with higher diagnostic yield [44], is an aerosolgenerating procedure used in selected patients (e.g. intubated patients) in order to minimise exposure of healthcare workers to SARS-CoV-2 [45]. Nevertheless, considering the false negative rate in COVID-19 pneumonia reported in previous studies $[46,47]$ and the number of SARS-CoV-2 negative patients with pneumonia in our study, a little proportion of patients were misclassified $(\approx 3.1 \%)$. According to the high sample size of the study, misclassification had slight effect on statistical estimations. Third, in nonCOVID-19 patients, the aetiology of the acute respiratory disease was not investigated. Patients with chronic comorbidities infected with nonSARS-CoV-2 respiratory viruses have a poorer prognosis $[48,49]$. Fourth, since we used an open-data dataset, adverse clinical outcomes could develop in patients after updating the web page; however, patients with and without comorbidities had the same chance to develop outcomes, with little or no impact on estimations. Fifth, we only used the chronic comorbidities recorded in the administrative healthcare open data; therefore other chronic underlying diseases likely associated with adverse clinical outcomes were not explored; for instances, malignancies, cerebrovascular diseases and liver diseases have also linked to poorer outcome in COVID-19 patients [3-5, 8]. Finally, the study was carried out in Mexican population, which could be a limitation for generalizing results. Because some limitations could affect statistical estimations, it should be considered before extrapolating results to a specific population. Overcoming these limitations is required for designing future researches.

\section{Conclusions}

Chronic underlying diseases are commonly found in patients with and without COVID-19, which are significant risk factors for adverse clinical outcomes. This knowledge can better define those COVID-19 patients at higher risk, who require more personalized and specific approaches to prevent complications. The different impact of the specific type of chronic comorbidity on clinical outcomes in patients with and without SARS-CoV-2 infection requires further researches. Although these findings need confirmation using other data sources, it is a useful contribution in our understanding of COVID-19.

Acknowledgments The authors thank Rosa M. Coro-Antich for their help in manuscript editing.

Author contributions All authors contributed to the study conception and design. Material preparation, data collection and formal analysis were performed by FDM-B. The first draft of the manuscript was written by FDM-B and all authors commented on previous versions of the manuscript. All authors read and approved the final manuscript.

Funding This research did not receive any specific grant from funding agencies in the public, commercial, or not-for-profit sectors.

Data availability All data generated or analysed during this study are included in this published article, and its supplementary information files and linked in reference 19.

Code availability As described in the text, a complete list of codes used to classify chronic comorbidities, outcomes, and confounders is provided in Online Resource 1.

\section{Compliance with ethical standards}

Conflict of interest The authors declared that they have no conflict of interest.

Human and animal rights The current study was conducted in accordance with the 1964 Helsinki Declaration and its later amendments or comparable ethical standards. The national administrative healthcare open data from the General Directorate of Epidemiology of Mexico was used; therefore the study was in accordance with the national ethical standard of Mexico. 
Informed consent Informed consent was waived in view of the retrospective nature of the study and all the procedures being performed were part of the routine care.

\section{References}

1. Huang C, Wang Y, Li X, Ren L, Zhao J, Hu Y et al (2020) Clinical features of patients infected with 2019 novel coronavirus in Wuhan, China. Lancet 395:497-506. https://doi.org/10.1016/ S0140-6736(20)30183-5

2. Wang C, Horby PW, Hayden FG, Gao GF (2020) A novel coronavirus outbreak of global health concern. Lancet 395:470-473. https://doi.org/10.1016/S0140-6736(20)30185-9

3. Emami A, Javanmardi F, Pirbonyeh N, Akbari A (2020) Prevalence of underlying diseases in hospitalized patients with COVID19: a systematic review and meta-analysis. Arch Acad Emerg Med 8:e35

4. Wang B, Li R, Lu Z, Huang Y (2020) Does comorbidity increase the risk of patients with COVID-19: evidence from meta-analysis. Aging (Albany NY) 12:6049-6057. https://doi.org/10.18632 laging. 103000

5. Hu Y, Sun J, Dai Z, Deng H, Li X, Huang Q et al (2020) Prevalence and severity of corona virus disease 2019 (COVID-19): a systematic review and meta-analysis. J Clin Virol 127:104371. https://doi.org/10.1016/j.jcv.2020.104371

6. Yang J, Zheng Y, Gou X, Pu K, Chen Z, Guo Q et al (2020) Prevalence of comorbidities and its effects in patients infected with SARS-CoV-2: a systematic review and meta-analysis. Int J Infect Dis 94:91-95. https://doi.org/10.1016/j.ijid.2020.03.017

7. Onder G, Rezza G, Brusaferro S (2020) Case-fatality rate and characteristics of patients dying in relation to COVID-19 in Italy. JAMA 323:1775-1776. https://doi.org/10.1001/jama.2020.4683

8. Guan WJ, Liang WH, Zhao Y, Liang HR, Chen ZS, Li YM et al (2020) Comorbidity and its impact on 1590 patients with Covid19 in China: a nationwide analysis. Eur Respir J 55:2000547. https ://doi.org/10.1183/13993003.00547-2020

9. Kumar A, Arora A, Sharma P, Anikhindi SA, Bansal N, Singla $V$ et al (2020) Is diabetes mellitus associated with mortality and severity of COVID-19? A meta-analysis. Diabetes Metab Syndr 14:535-545. https://doi.org/10.1016/j.dsx.2020.04.044

10. Tamara A, Tahapary DL (2020) Obesity as a predictor for a poor prognosis of COVID-19: a systematic review. Diabetes Metab Syndr 14:655-659. https://doi.org/10.1016/j.dsx.2020.05.020

11. Alqahtani JS, Oyelade T, Aldhahir AM, Alghamdi SM, Almehmadi M, Alqahtani AS et al (2020) Prevalence, severity and mortality associated with COPD and smoking in patients with COVID-19: a rapid systematic review and meta-analysis. PLoS ONE 15:e0233147. https://doi.org/10.1371/journal.pone.0233147

12. General Directorate of Epidemiology of Mexico (2020) Open data. https://www.gob.mx/salud/documentos/datos-abiertos152127. Accessed 13 May 2020

13. Institute of Epidemiological Diagnosis and Reference "Dr Manuel Martínez Báez" (2020) https://www.gob.mx/salud/acciones-yprogramas/instituto-de-diagnostico-y-referencia-epidemiologicos -indre. Accessed 01 June 2020

14. Corman VM, Landt O, Kaiser M, Molenkamp R, Meijer A, Chu DK et al (2020) Detection of 2019 novel coronavirus (2019nCoV) by real-time RT-PCR. Euro Surveill 25:2000045. https ://doi.org/10.2807/1560-7917.ES.2020.25.3.2000045

15. Johns Hopkins University and Medicine (2020) Coronavirus Resource Center: new cases of COVID-19 in world countries. https://coronavirus.jhu.edu/data/new-cases. Accessed 07 Oct 2020

16. Grasselli G, Zangrillo A, Zanella A, Antonelli M, Cabrini L, Castelli A et al (2020) Baseline characteristics and outcomes of 1591 patients infected with SARS-CoV-2 admitted to ICUs of the Lombardy region. Italy JAMA 323:1574-1581. https://doi. org/10.1001/jama.2020.5394

17. Guan W, Ni Z, Hu Y, Liang W, Ou C, He J et al (2020) Clinical characteristics of coronavirus disease 2019 in China. N Engl J Med 382:1708-1720. https://doi.org/10.1056/NEJMoa2002032

18. Zhou F, Yu T, Du R, Fan G, Liu Y, Liu Z et al (2020) Clinical course and risk factors for mortality of adult inpatients with COVID-19 in Wuhan, China: a retrospective cohort study. Lancet 395:1054-1062. https://doi.org/10.1016/S0140-6736(20)30566-3

19. Zheng Z, Peng F, Xu B, Zhao J, Liu H, Peng J et al (2020) Risk factors of critical \& mortal COVID-19 cases: a systematic literature review and meta-analysis. J Infect 81:e16-e25. https://doi. org/10.1016/j.jinf.2020.04.021

20. Felsenstein S, Herbert JA, McNamara PS, Hedrich CM (2020) COVID-19: immunology and treatment options. Clin Immunol 215:108448. https://doi.org/10.1016/j.clim.2020.108448

21. Del Sole F, Farcomeni A, Loffredo L, Carnevale R, Menichelli D, Vicario $T$ et al (2020) Features of severe COVID-19: a systematic review and meta-analysis. Eur J Clin Invest 50:e13378. https://doi. org/10.1111/eci.13378

22. Klein SL, Flanagan KL (2016) Sex differences in immune responses. Nat Rev Immunol 16:626-638. https://doi.org/10.1038/ nri.2016.90

23. Farsalinos K, Barbouni A, Poulas K, Polosa R, Caponnetto P, Niaura R (2020) Current smoking, former smoking, and adverse outcome among hospitalized COVID-19 patients: a systematic review and meta-analysis. Ther Adv Chronic Dis 11:2040622320935765. https://doi.org/10.1177/2040622320 935765

24. González-Rubio J, Navarro-López C, López-Nájera E, LópezNájera A, Jiménez-Díaz L, Navarro-López JD et al (2020) A systematic review and meta-analysis of hospitalised current smokers and COVID-19. Int J Environ Res Public Health 17:7394. https:// doi.org/10.3390/ijerph17207394

25. Polosa R, Caci G (2020) COVID-19: counter-intuitive data on smoking prevalence and therapeutic implications for nicotine. Intern Emerg Med 15:853-856. https://doi.org/10.1007/s1173 9-020-02361-9

26. Sanchez-Ramirez DC, Mackey D (2020) Underlying respiratory diseases, specifically COPD, and smoking are associated with severe COVID-19 outcomes: A systematic review and meta-analysis. Medicine 171:106096. https://doi.org/10.1016/j. rmed.2020.106096

27. Gülsen A, Yigitbas BA, Uslu B, Drömann D, Kilinc O (2020) The effect of smoking on COVID-19 symptom severity: systematic review and meta-analysis. Pulm Med 2020:7590207. https://doi. org/10.1155/2020/7590207

28. Salah HM, Sharma T, Mehta J (2020) Smoking doubles the mortality risk in COVID-19: a meta-analysis of recent reports and potential mechanisms. Cureus 12:e10837. https://doi.org/10.7759/ cureus. 10837

29. Cai G, Bossé Y, Xiao F, Kheradmand F, Amos CI (2020) Tobacco smoking increases the lung gene expression of ACE2, the receptor of SARS-CoV-2. Am J Respir Crit Care Med 201:1557-1559. https://doi.org/10.1164/rccm.202003-0693LE

30. Hales K, Carroll MD, Fryar CD, Ogden CL (2020) Prevalence of obesity and severe obesity among adults: United States, 20172018. NCHS Data Brief; 360:1-8. https://www.cdc.gov/nchs/ products/databriefs/db360.htm. Accessed 20 Nov 2020 
31. Hussain A, Mahawar K, Xia Z, Yang W, El-Hasani S (2020) Obesity and mortality of COVID-19. Meta-analysis. Obes Res Clin Pract 14:295-300. https://doi.org/10.1016/j.orcp.2020.07.002

32. Popkin BM, Du S, Green WD, Beck MA, Algaith T, Herbst CH et al (2020) Individuals with obesity and COVID-19: a global perspective on the epidemiology and biological relationships. Obes Rev 21:e13128. https://doi.org/10.1111/obr.13128

33. Caci G, Albini A, Malerba M, Noonan DM, Pochetti P, Polosa R (2020) COVID-19 and obesity: dangerous liaisons. J Clin Med 9:2511. https://doi.org/10.3390/jcm9082511

34. Korean Society of Infectious Diseases; Korean Society of Pediatric Infectious Diseases; Korean Society of Epidemiology; Korean Society for Antimicrobial Therapy; Korean Society for Healthcare-associated Infection Control and Prevention; Korea Centers for Disease Control and Prevention (2020) Report on the epidemiological features of coronavirus disease 2019 (COVID-10) outbreak in the Republic of Korea from January 19 to March 2, 2020. J Korean Med Sci 35:e112. https://doi.org/10.3346/jkms.2020.35. e112

35. Li X, Xu S, Yu M, Wang K, Tao Y, Zhou Y et al (2020) Risk factors for severity and mortality in adult COVID-19 inpatients in Wuhan. J Allergy Clin Immunol 146:110-118. https://doi. org/10.1016/j.jaci.2020.04.006

36. Paranjpe I, Russak AJ, De Freitas JK, Lala A, Miotto R, Vaid A (2020) Clinical characteristics of hospitalized Covid-19 patients in New York City. medRxiv. https://doi. org/10.1101/2020.04.19.20062117

37. Liu S, Zhi Y, Ying S (2020) COVID-19 and asthma: reflection during the pandemic. Clin Rev Allergy Immunol 59:78-88. https ://doi.org/10.1007/s12016-020-08797-3

38. Chen X, Zhao B, Qu Y, Chen Y, Xiong J, Feng, et al (2020) Detectable serum SARS-CoV-2 viral load (RNAaemia) is closely correlated with drastically elevated interleukin 6 (IL-6) level in critically ill COVID-19 patients. Clin Infect Dis. https://doi. org/10.1093/cid/ciaa449

39. Shlomai A, Ben-Zvi H, Bendersky AG, Shafran N, Goldberg E (2020) Sklan EH (2020) Nasopharyngeal viral load predicts hypoxemia and disease outcome in admitted COVID-19 patients. Crit Care 24:539. https://doi.org/10.1186/s13054-020-03244-3

40. de Lucena TMC, da Silva Santos AF, de Lima BR, de Albuquerque Borborema ME, de Azevedo SJ (2020) Mechanism of inflammatory response in associated comorbidities in COVID-19.
Diabetes Metab Syndr 14:597-600. https://doi.org/10.1016/j. dsx.2020.05.025

41. Li Y, Zhou W, Yang L, You R (2020) Physiological and pathological regulation of ACE2, the SARS-CoV-2 receptor. Pharmacol Res 157:104833. https://doi.org/10.1016/j.phrs.2020.104833

42. Pons S, Fodil S, Azoulay E, Zafrani L (2020) The vascular endothelium: the cornerstone of organ dysfunction in severe SARS-CoV-2 infection. Crit Care 24:353. https://doi.org/10.1186/ s13054-020-03062-7

43. The Severe Covid-19 GWAS Group (2020) Genomewide Association Study of severe Covid-19 with respiratory failure. N Engl J Med. https://doi.org/10.1056/NEJMoa2020283

44. Wang W, Xu Y, Gao R, Lu R, Han K, Wu G et al (2020) Detection of SARS-CoV-2 in different types of clinical specimens. JAMA 323:1843-1844. https://doi.org/10.1001/jama.2020.3786

45. Wahidi MM, Shojaee S, Lamb CR, Ost D, Maldonado F, Eapen $\mathrm{G}$ et al (2020) The Use of bronchoscopy during the Coronavirus Disease 2019 pandemic: CHEST/AABIP guideline and expert panel report. Chest 158:1268-1281. https://doi.org/10.1016/j. chest.2020.04.036

46. Ai T, Yang Z, Hou H, Zhan C, Chen C, Lv W et al (2020) Correlation of chest $\mathrm{CT}$ and RT-PCR testing in coronavirus disease 2019 (COVID-19) in China: a report of 1014 cases. Radiology 296:E32-E40. https://doi.org/10.1148/radiol.2020200642

47. Li Y, Yao L, Li J, Chen L, Song Y, Cai Z et al (2020) Stability issues of RT-PCR testing of SARS-CoV-2 for hospitalized patients clinically diagnosed with COVID-19. J Med Virol 92:903-908. https://doi.org/10.1002/jmv.25786

48. Matsuyama R, Nishiura H, Kutsuna S, Hayakawa K, Ohmagari N (2016) Clinical determinants of the severity of Middle East respiratory syndrome (MERS): a systematic review and metaanalysis. BMC Public Health 16:1203. https://doi.org/10.1186/ s12889-016-3881-4

49. Gounder AP, Boon ACM (2019) Influenza Pathogenesis: The role of host factors on severity of disease. J Immunol 202:341-350. https://doi.org/10.4049/jimmunol.1801010

Publisher's Note Springer Nature remains neutral with regard to jurisdictional claims in published maps and institutional affiliations. 